\title{
A novel aerosolisation mitigation device for endoscopic sinus and skull base surgery in the COVID-19 era
}

\author{
Jaime Viera-Artiles ${ }^{1}$ (1) $\cdot$ David Mato $^{2}$ • José J. Valdiande ${ }^{3}$. David Lobo ${ }^{1} \cdot$ Juan Pedraja ${ }^{4}$. José M. López-Higuera ${ }^{3,5}$. \\ Javier Ospina ${ }^{6}$
}

Received: 11 September 2020 / Accepted: 28 October 2020 / Published online: 16 November 2020

(c) Springer-Verlag GmbH Germany, part of Springer Nature 2020

\begin{abstract}
Purpose To provide a novel solution to reduce aerosol exposure in the operating room during endoscopic sinus and skull base procedures in the COVID-19 era.

Methods We have designed a 3D printable midfacial mask that partially seals the nose, while allowing instrumentation during endoscopic transnasal surgery. The mask when connected to a vacuum system creates a constant negative pressure inside it, sucking out aerosols and gases generated during surgical procedures. Its effectiveness was tested using vapour exhalations by a human volunteer and drilling bone in a head model. The physical barrier effect was measured using fluorescein atomization in a head model.

Results The pressure and airflow measured remained negative inside it in all the different situations tested. The mask was capable of completely evacuating human adult exhalation, and was more effective than the hand suction instrument. However, it was as effective as hand suction instrument at preventing aerosol spread from bone drilling. The physical barrier effect achieved a $72 \%$ reduction in the splatter created from the fluorescein atomization.

Conclusions The mask effectively prevented the spread of aerosols and reduced droplet spread during simulated transnasal endoscopic skull base surgery in laboratory conditions. This device has potential benefits in protecting surgical personnel against airborne transmission of COVID-19 and could be useful in reducing chronic exposure to the hazard of surgical smoke.
\end{abstract}

Keywords SARS-CoV-2 $\cdot$ COVID-19 $\cdot$ Endoscopic sinus surgery $\cdot$ Skull base surgery $\cdot$ Infection prevention

Electronic supplementary material The online version of this article (https://doi.org/10.1007/s00405-020-06462-1) contains supplementary material, which is available to authorized users.

Jaime Viera-Artiles

jaimevieraartiles@gmail.com

1 ENT Department, Hospital Universitario Marqués de Valdecilla, Santander, Spain

2 Neurosurgery Department, Hospital Universitario Marqués de Valdecilla, Santander, Spain

3 Photonics Engineering Group, Universidad de Cantabria, Santander, Spain

4 Hospital Virtual de Valdecilla, Santander, Spain

5 Centro de Investigacion Biomédica En Red (CIBER-BBN), Instituto De Investigación Marqués de Valdecilla (IDIVAL), Santander, Spain

6 Division of Otolaryngology - Sinus and Skull Base Surgery, Fundación Santa Fe de Bogotá, Bogotá, Colombia

\section{Introduction}

Coronavirus disease 2019 (COVID-19) resulting from the novel coronavirus strain (SARS-CoV-2) represents an extraordinary threat to the health of the world's population. Different modes of transmission have been described, and all contribute to the exponential growth of the virus worldwide [1]. The major mode of transmission is thought to occur via direct contact or large respiratory droplets. Owing to their weight and size (larger than 20 microns), these droplets travel only a short distance before settling on surfaces. However, there is also a risk of virus spread in smaller aerosols generated during various medical procedures that can cause airborne transmission over extensive distances [2-5]. Aerosol-generating medical procedures have been recognised as an important source for nosocomial transmission of emerging viruses [6-8]. It has been observed that once aerosolized, SARS-CoV-2 particles may remain viable and infectious in the air for at least $3 \mathrm{~h} \mathrm{[4].}$ 
Recently, Zou et al. showed that the viral load of SARSCoV-2 is higher in the nasal cavity than in the throat, regardless of whether the patient is symptomatic or not [9]. Hence, this is of concern for nasal endoscopy, and even more so for endoscopic endonasal skull base surgery [10], since during this procedure many mechanisms can result in the aerosolization of mucus and virus which may permeate the operating environment. Importantly, aerosol formation during procedures may be divided into patient induced or mechanically induced. Since endonasal surgery is carried out in the setting of an occluded lower airway, the risk of aerosol generation, particle size, and transmission distance are entirely a function of the instrumentation utilised, and are, therefore, mechanically induced. Finally, it should be pointed out that mechanical procedures may be divided into thermal and cold procedures, with the latter further divided into powered and non-powered [11].

During the evolving COVID-19 pandemic, cadaveric studies that simulated surgical conditions confirmed the potential infectious aerosolization patterns resulting from mechanical-cold procedures, especially powered procedures $[11,12]$. These studies proposed possible mitigation strategies such as mechanical barriers (e.g. modified standard surgical mask), or the use of concurrent suction in the anterior nasal cavity, both of which greatly reduce the risk of environmental contamination. Although none of these investigations analysed aerosol generation during thermal procedures, which occurs frequently during endoscopic sinus and skull base surgery (e.g. nasoseptal flap harvesting technique) numerous articles suggest that these procedures do have the potential to generate virus-laden aerosols, such as surgical smoke or vapour, and should be approached with caution [13-16]. Active aspiration systems during thermal procedures have been recommended to reduce smoke dispersion, and consequently the hazard of infection [13].

Under these circumstances, it is essential that innovators, inventors and surgeons come together to develop novel strategies to alleviate such high-risk factors. For instance, makers and designers in the 3D printing community have responded to the global crisis by volunteering their respective skills to ease the pressure on supply chains and governments. Thus, 3D printing techniques are becoming essential in this context, especially by increasing the availability of respirators and providing personal protective equipment [17, 18]. The main advantages of this technology are their current widespread distribution and the low associated costs, which allow new designs to be readily available worldwide in a matter of hours.

Since the use of mechanical barriers and continuous active suction during endoscopic sinus and skull base surgery is considered an effective method to reduce potential infectious aerosols, which is especially important during the COVID-19 pandemic, we have designed a 3D printable midfacial mask with a suction port that generates a constant negative pressure inside to suck out aerosols and gases while permitting comfortable nasal and skull base instrumentation. The mask, which is closed anteriorly by a flexible valve through which surgical instruments are introduced, aims to effectively reduce exposure to respiratory droplets during endoscopic sinus and skull base procedures. This article describes this novel device named the "Maskpirator" and analyses its properties and effectiveness under laboratory conditions.

\section{Materials and methods}

\section{Mask design}

The 3D model for the mask was designed with DesignSpark Mechanical CAD/CAM software (Ansys, Inc., Canonsburg, PA, USA) and the prototype was printed with a stereolithography (SLA) 3D printer in white resin (Formlabs, Somerville, MA, USA), a biocompatible, sterilisable and reusable material.

The Maskpirator is ergonomically designed to create a partially sealed midfacial chamber above the nose (Fig. 1). The mask includes two ports with different functions. A work port is located anteriorly and its size and angulation are designed to comfortably allow the passage of endoscopes and surgical tools during surgery (Fig. 1, white arrow); a suction port is located superiorly and allows the connection of a tube to a vacuum system without interfering with the working port (Fig. 1, arrowhead). The purpose of this second port is to create a constant negative pressure inside the mask to suck out aerosols and gases generated during nasal manipulation. The mask can be held to the patient's head with elastic bands, for instance, that can be attached to the handles located on the sides of the mask (Fig. 1, black arrow).

To cover the work port during surgical interventions, we have designed a thin $(0.8 \mathrm{~mm})$ elastic valve that allows multiple tool manipulation simultaneously (Fig. 1c). The material used for this part was a thermoelastic elastomer (Formlabs elastic resin), a single-use, flexible material printed with an SLA 3D printer. Furthermore, this port has a thick rim which accommodates a surgical glove and creates an airtight seal when used with an endoscope (rigid or flexible) (Fig. 1f). This is a simple solution made from readily available material that could be used for nasal endoscopic examination.

\section{Effectiveness evaluation}

The Maskpirator's effectiveness at evacuating aerosols and preventing droplet spread was tested in several experiments 


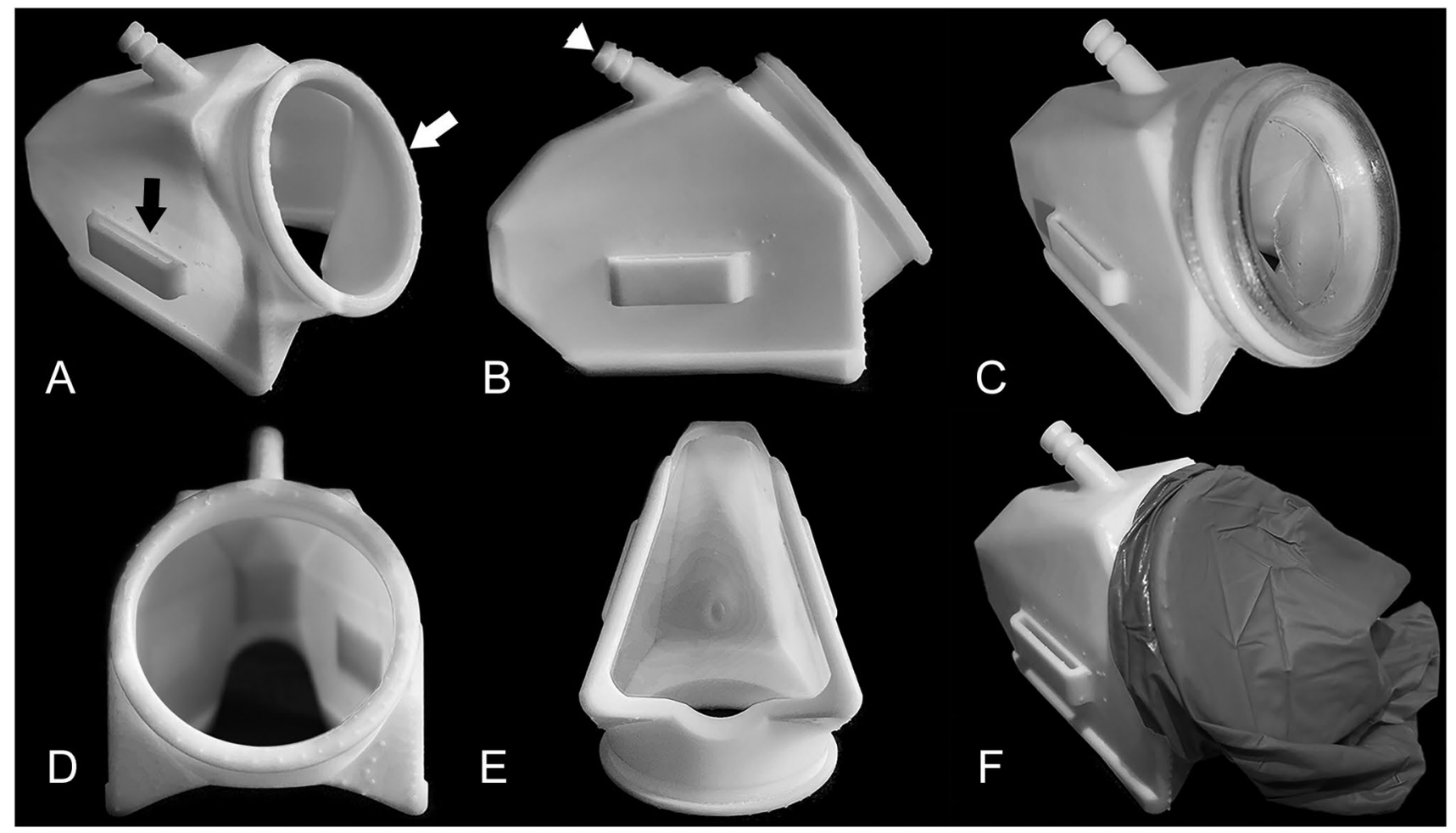

Fig. 1 The final prototype printed in white resin. The working port (white arrow) is placed anteriorly, the suction port (arrow head) superiorly and handles on both sides (black arrow). a Perspective view. b

performed in a surgical simulation laboratory in "Hospital Virtual Valdecilla" in Santander, Spain.

First, we measured the mask's properties using specialised airflow and pressure measuring tools. Second, to visualise and video record the airflow dynamics outside the mask, we carried out two different sets of empirical experiments through vapour exhalations from a human volunteer and by drilling bone inside a head model. Finally, the reduction of droplets spread was evaluated in another experiment using atomized fluorescein in a head model.

\section{Aerodynamic properties of the mask}

Two different physical parameters were measured: pressure and airflow. The pressure inside the mask with and without the valve cover attached to the work port was measured with a digital manometer Hti HT-1980 to determine the differential pressure at two different points: the aspiration tube outlet and inside the mask's chamber, in front of the nasal tip.

Second, to calculate the airflow entering the mask's central chamber and counteracting the volunteer's exhalation when the vacuum suction is connected, we constructed a specific setup (Fig. 2). Considering that the mask is airtight with only two openings (the aspiration
Lateral view. c Maskpirator with the valve cover. d Frontal view. e Inferior view. f Maskpirator with glove cover

tube connected to the vacuum system and the work port with the valve cover at the front), the total airflow balance at the front entrance can be calculated by subtracting the exhalation airflow from the aspiration airflow. The airflow at the mask's work port was obtained by measuring the air velocity $(\mathrm{m} / \mathrm{s})$ with a hot wire anemometer KIMO CTV100 (Fig. 2, white arrow) connected to an analogue-to-digital acquisition system inside a $58 \mathrm{~mm}$ diameter cylinder with an equivalent free open area of $2122 \mathrm{~mm}^{2}$ (Fig. 2, black arrow), and the cylinder attached to the work port (with the valve cover), in three different situations: (a) measuring the volunteer's exhalation airflow with the mask in position without a vacuum; (b) measuring the airflow with the mask in position and the vacuum suction activated, but the volunteer in apnoea; and (c) measuring the volunteer's exhalation airflow with the mask in position and the vacuum system connected.

These values were then transformed to litres/minute by applying a conversion constant using the velocity and the cross-section area of the work port cylinder. The data were obtained measuring during one second, with a sample rate of $0.5 \mathrm{~ms}$; therefore, 2000 samples per experiment were obtained. 
Fig. 2 Setup to calculate the airflow in the mask, consistent of a hot wire anemometer KIMO CTV100 (white arrow) and an analogue-to-digital acquisition system, inside a $58 \mathrm{~mm}$ diameter cylinder connected to the mask (black arrow)

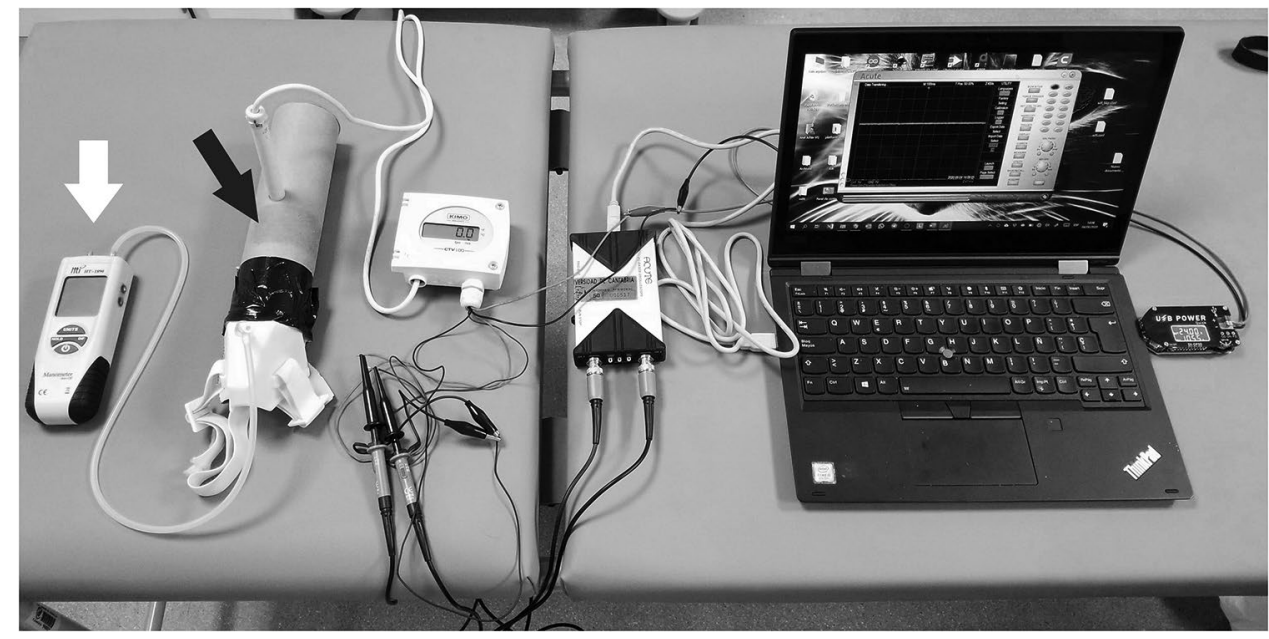

\section{Visual experiments}

Vapour exhalations To visualise the mask's effectiveness at preventing the spread of aerosols and gases, we performed a series of experiments using vapour exhalations from an electronic cigarette. The mask was also compared to hand suction.

Experiment 1 A human volunteer wearing the mask inhaled and exhaled the same amount of vapour (approx. $3500 \mathrm{ml}$ ). Then, we performed the tests in the following three situations: (a) without vacuum suction; (b) with the suction connected to a vacuum system; (c) with the working port partially sealed by the valve cover.

Experiment 2 The same volunteer exhaled vapour in another three different situations: (a) with the mask in position and two instruments inserted in the work port through the flexible valve; (b) similar to the previous experiment and the mask connected to a vacuum suction system; and (c) similar to experiment $2 \mathrm{a}$ but with the vacuum system connected to a hand suction instrument. Experiments $2 \mathrm{a}$ and $2 \mathrm{~b}$ were repeated in 6 volunteers ( 4 males and 2 females).

Bone drilling A S.I.M.O.N.T. head model for ENT and skull base surgery (KARL STORZ, Tuttlingen, Germany) was used to perform a bilateral sphenoid sinusotomy and sphenoid rostrectomy. For a simulation of real bone drilling, customised temporal bone blocks were placed inside the sphenoid and drilled, as in the operating room, using a MIDAS high-speed surgical drill (Medtronic, MI, USA) with a $4 \mathrm{~mm}$ diamond burr at $60,000 \mathrm{rpm}$.

Experiment 3 The drilling was tested using the mask in position over the head model face in the following situations: (a) without vacuum suction; (b) with the vacuum suction connected; and (c) with the vacuum suction connected to a hand suction system instead of the mask.

Prevention of droplet spread The Maskpirator's effectiveness at preventing droplet spread was tested on a rubber head model with an upper airway. A nasal mucosal atomization device (Mad Nasal, Teleflex Medical, Morrisville, NC, USA) that produces particles between $30 \mu \mathrm{m}$ and $100 \mu \mathrm{m}$ in size was placed in the left nostril and connected by an extension tube to a $5 \mathrm{cc}$ syringe filled with a mixture consisting of fluorescein and saline solution. After the experiments, all the elements involved were inspected under a $15 \mathrm{~W} 365 \mathrm{~nm}$ UV light lamp to identify the fluorescence droplets.

Experiment 4 For a simulation of surgical conditions, the head model was placed horizontally inside a sealed methacrylate box, with the inner superior surface $(50 \times 35 \mathrm{~cm})$ divided into six quadrants $(12.5 \times 17.5 \mathrm{~cm})$ and separated $8 \mathrm{~cm}$ from the nose. The solution was atomized with a $5 \mathrm{cc}$ syringe plunged at maximum pressure under two different conditions: (a) the head model without any barriers; (b) the head model with the Maskpirator covering the nose, the working port sealed by the valve cover pierced by a $4 \mathrm{~mm}$ endoscope, the endoscope inserted inside the right nasal fossa, and a vacuum suction system connected to the suction port.

To test this proof of concept, each experiment was only performed once. The extent of the droplet splatter generated in each condition was analysed using images of the inner surface and SketchAndCalc ${ }^{\mathrm{TM}}$ area calculation software (Palm Coast, FL, USA). 


\section{Results}

\section{Mask design}

The estimated production cost for one mask is approximately $\$ 15 \mathrm{US}$, and the printing time is $<5 \mathrm{~h}$. The prototype perfectly adapted to the volunteer's face and was comfortable enough to be used during all the experiments with no pressure damage to the skin.

\section{Aerodynamic properties of the mask}

Pressure The pressure in the suction outlet port with the mask in position and connected to the hospital's vacuum system was $-41 \mathrm{mBar}$. The hospital's vacuum system provides a suction pressure of $-240 \mathrm{mBar}$.

The pressure at the nasal tip with the mask in position and the valve cover placed correctly was $-0.5 \mathrm{mBar}$, which varied from $-0.4 \mathrm{mBar}$ when the volunteer exhaled to - $0.6 \mathrm{mBar}$ on inhalation.

Airflow The absolute values in litres/minute obtained in the three different situations tested were the following:

- Situation a (volunteer's exhalation): 9.04 1/min (range $6.0-11.131 / \mathrm{min})$.

- Situation b (vacuum activated): 28.82 1/min (range 24.81-33.36 1/min).

- Situation c (exhalation and vacuum): 23.32 1/min (range 21.39-26.52 1/min).

\section{Visual experiments}

Vapour exhalations Experiment 1 (Video 1) The reduction in vapour spread is visible when comparing the mask without suction to the mask with the vacuum suction connected (Figs. 3a and 1b). Turbulence and airflow inversion can be seen at the periphery of the work port, with some vapour escaping into the environment when the port is not covered by the valve (Fig. 3b). However, in experiment 1c, when the valve cover is in position and partially sealing the work port, there is no escape of vapour (Fig. 3c).

Experiment 2 In a more realistic setting, with two instruments inserted through the valve cover, there is still no vapour leakage when the suction is connected to the mask (Video 2). In experiment 2b (Video 3), in the comparison with hand suction, the mask proved to be more effective at preventing the escape of vapour (Fig. 4).

The result of the experiments $2 \mathrm{a}$ and $2 \mathrm{~b}$ was similar in the six volunteers, with no visible vapour leakage when the suction is connected to the mask.

Bone drilling Experiment 3 To generate enough bone dust to be visible and recorded escaping from the nose, a substantial amount of temporal bone needed to be drilled without irrigation (Video 4). In these experiments, both the mask and the hand suction were equally effective in preventing the bone dust from escaping into the environment (Video 5). Prevention of droplet spread The experiment with the head model in the box without the mask (condition 4a) resulted in droplet and aerosol splatter over the central quadrants of the inner superior surface covering an area of $298.86 \mathrm{~cm}^{2}$. In condition $4 \mathrm{~b}$, using the valve cover, the splatter area extended over $86.06 \mathrm{~cm}^{2}$, which was $72 \%$ smaller than the splatter formed without the mask (Fig. 5).

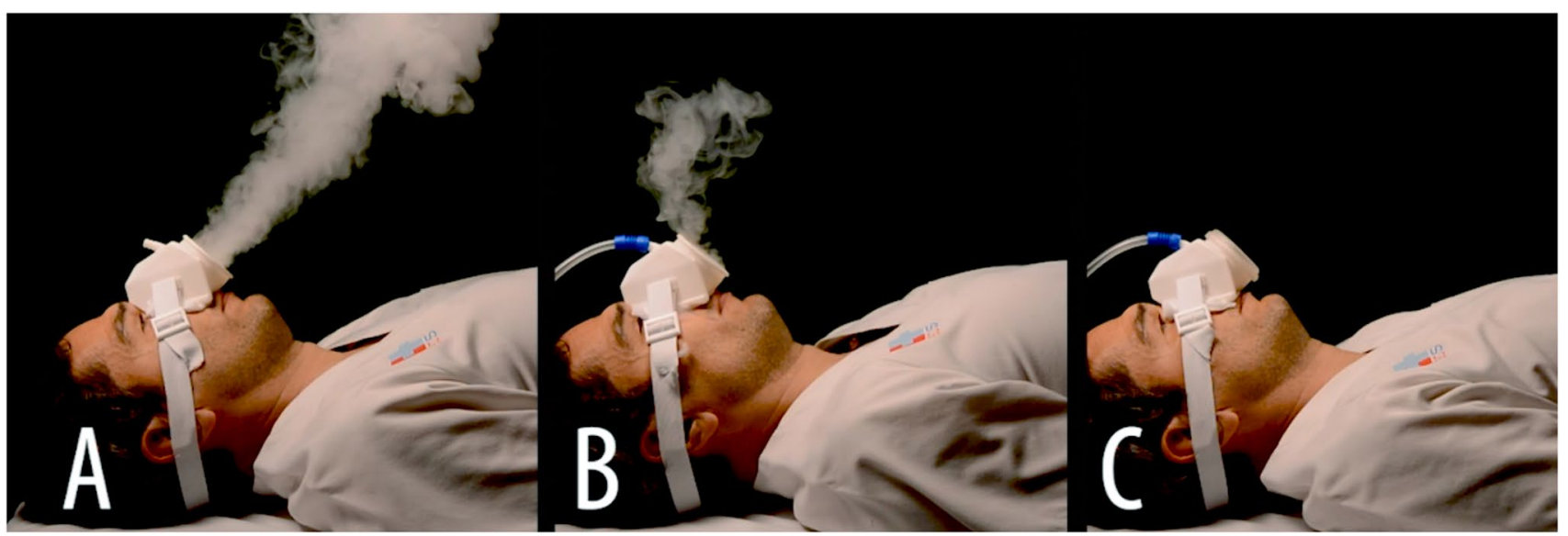

Fig. 3 Experiment 1 after $2 \mathrm{~s}$ of exhaling vapour in the following three situations: a without vacuum suction; $\mathbf{b}$ with the suction connected to a vacuum system; $\mathbf{c}$ with the working port partially sealed by the valve cover 


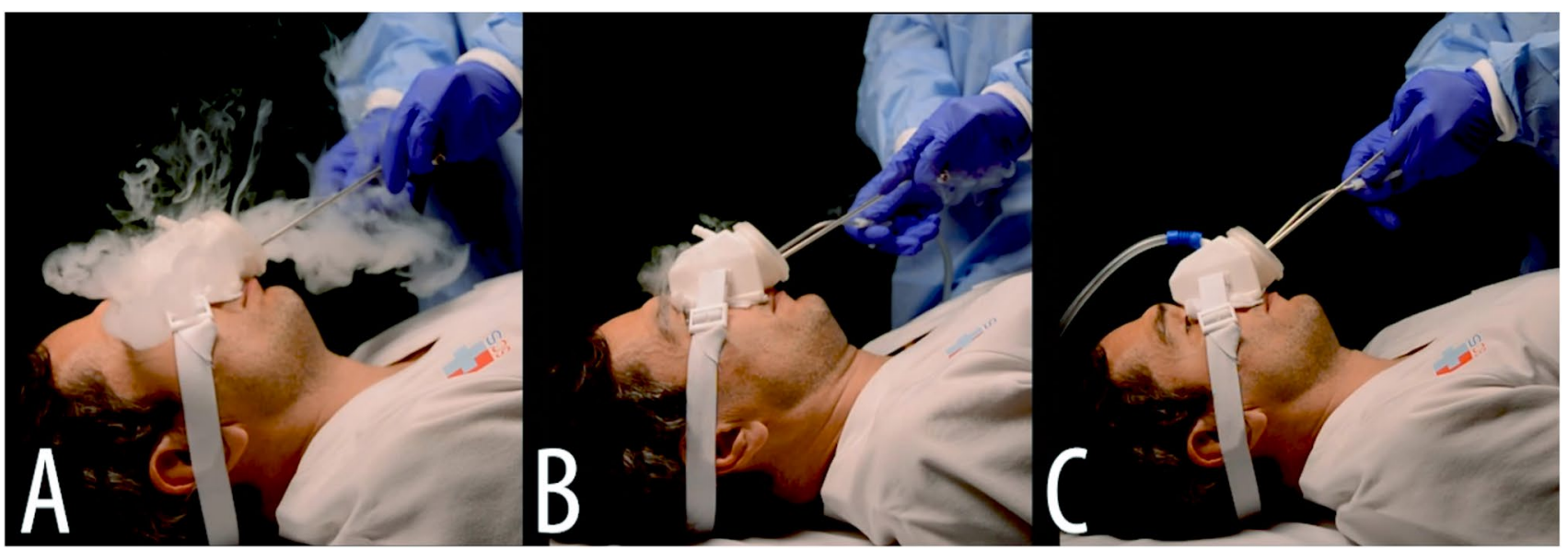

Fig. 4 Experiment 2 after $2 \mathrm{~s}$ of exhaling vapour in the following three situations: a with the mask in position and two instruments inserted in the work port through the flexible valve $\mathbf{b}$ similar to the previous experiment and the mask connected to a vacuum suction system. c Similar to experiment 2a but with the vacuum system connected to a hand suction instrument

\section{Discussion}

The novel coronavirus disease (COVID-19) caused by the SARS-CoV 2 virus was designated a pandemic on 11 March 2020 and has rapidly spread worldwide, affecting more than 20 million people, a figure which is, unfortunately, increasing every day. Healthcare providers are at the front line of the response to the pandemic and as such are exposed to several hazards that put them at risk of infection. Physicians and surgeons must assume responsibility to ensure that all necessary preventive and protective measures are taken to minimise occupational safety and health risks $[19,20]$. There is increasing concern among sinus and skull base surgeons in the neurosurgery and otolaryngology community due to exposure to a high viral load reservoir during endoscopic procedures in the nasal cavity and the nasopharynx [21].

Workman et al. have recently published their effort to simulate nasal aerosolisation during a variety of endonasal procedures and proposed different potential mitigation strategies. They described the valved endoscopy of the nose and throat (VENT) mask, where they customised a standard surgical mask with a central perforation covered with a piece of a non-latex glove to allow the passage of an endoscope [11]. This modification is meant to be used in conscious patients and it does not allow adequate instrumentation during complex interventions when the surgeon needs to constantly change instruments; it is not, therefore, suitable for endoscopic sinus and anterior skull base surgery.

Maskpirator offers several advantages. First, as demonstrated in our experiments, it reduces the possibility of aerosol leakage and droplet spread during endoscopic endonasal skull base procedures by providing partial sealing of the nasal cavity with continuous suction. Second, the design of this device permits comfortable manipulation of instruments, especially when they need to be replaced constantly, which potentially allows surgeons to perform complex interventions, such as endoscopic sinus and skull base procedures, with increased safety. Third, this device can be easily printed in a $3 \mathrm{D}$ printer machine and does not require special materials for its manufacture. The model is also scalable to fit different face sizes and can be upgraded and modified. Depending on the material used for printing, it can be sterilisable and reusable. However, sterilisation details should be individualised after consulting the manufacturer of the material.

To prevent gases from the nasal fossa escaping into the environment, the aspiration airflow inside the mask must be greater than exhalation airflow. With the setup described, our result for aspiration airflow was about 2.5 times greater than exhalation airflow. Therefore, the vacuum applied to the mask seems sufficient to prevent gas or aerosol leakages.

Additionally, the "negative pressure" values obtained inside the mask's chamber and at the vacuum port ("pressure" means relative pressure versus atmospheric pressure outside the mask) indicate that the air is always flowing from outside the mask into the vacuum port, through the valve in the frontal opening.

The consistency of data obtained in experiment $2 a$ and $2 b$ in the six volunteers indicates that the ability of the mask to reduce vapour leakage does not depend on the patient's facial anatomy and proportions. These results might be explained by the constant negative pressure inside the mask and the aspiration airflow.

In video $1 \mathrm{~b}$, turbulence and swirls can be seen to occur at the periphery of the work port. These effects enable portions of the exhaled vapour to leakage of the mask and are caused because the nose is close to the mask's entrance. However, in experiment $1 \mathrm{c}$, the placement of the valve cover in the work 
Fig. 5 Setup to estimate droplet spread prevention in experiment 4. a The head model inside the box for the experiment under condition A. b The fluorescein splatter over the central quadrants of the superior inner surface, after the experiment in A, using the UV light lamp. c The setup for condition $\mathrm{B}$, using a thicker cylinder to simulate two instruments. $\mathbf{d}$ The fluorescein splatter after experiment in C, without the UV light lamp

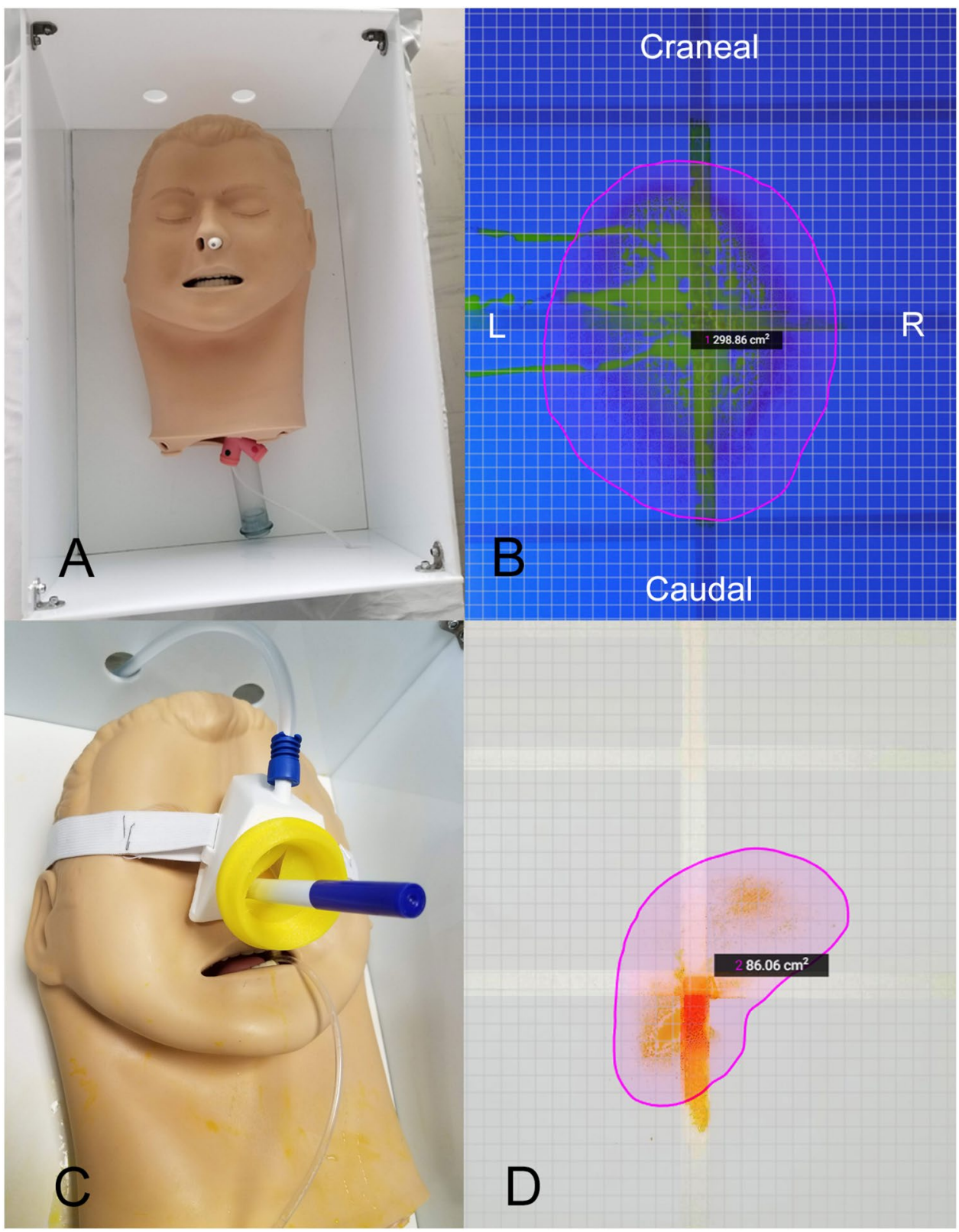

port eliminates turbulence and swirls at the periphery of the mask, preventing any vapour leakage. To study these effects properly, computational fluid dynamics (CFD) simulation software might be used and the airflow and pressure values obtained in our experiments are essential to perform a CFD analysis. This technique could be used to optimise the design of this prototype for different situations and applications; however, this is beyond the scope of this study.

When the endoscope and surgical tools were inserted into the nasal cavity through the valve cover (Experiment 2), the valve was wide open and, consequently, the seal was not airtight. Nonetheless, there was still no leakage in any of the experiments performed. This can be explained by the negative pressure generated inside the mask when connected to the vacuum system and the prevention of turbulence when the valve cover was used. The cover also provides certain protection from droplets, as demonstrated in experiment 4 , which could be useful when drilling and during tool manipulation.

The mask was designed for use in surgeries under general anaesthesia where the lower airway is usually blocked. However, the negative pressure generated inside the mask was effective enough to prevent vapour leakage from a conscious volunteer's exhalation. Therefore, there is potential use in such patients, for example when performing in-office nasal endoscopic procedures or examinations.

Our experiments have demonstrated that the mask is effective for retaining even large quantities of gases and 
aerosols in laboratory conditions, which could be extrapolated to real surgeries. Moreover, the mask proved more effective than hand suction in the vapour exhalations experiment, which simulated surgical smoke and other aerosol leakage. Nevertheless, it is unlikely that such a large amount of surgical smoke (approx. $3500 \mathrm{ml}$ in our experiments) would be generated in a real surgery and probably, hand suction would be enough to evacuate the smoke generated during a routine endoscopic transnasal skull base procedure (for example, in the course of bipolar cauterization of the dura or monopolar cauterization of bleeding nasal mucosa), as evidenced in the bone drilling experiments, where hand suction was enough to evacuate all the bone dust generated. However, if the hand suction instrument becomes clogged or if it is out of the nasal cavity at a certain point, the mask would provide extra protection that would rarely fail.

The surgical smoke released into the operating room environment when diathermy devices, such as an electrocauterization instrument, are used can cause adverse effects on health [22]. Although long-term effects have not been adequately studied, there is an increasing need to reduce or eliminate this smoke from the operating room and the Maskpirator could be useful for this purpose [23, 24].

There is currently no evidence that COVID-19 is transmissible through surgical smoke. Nevertheless, previous studies have demonstrated the presence of different viruses in surgical smoke, including corynebacterium, human papillomavirus (HPV), poliovirus, human immunodeficiency virus (HIV) and hepatitis B. This is another important reason for reducing the leakage of surgical smoke in the operating room [25].

Some limitations could be found derived from the mask's design. First, the mask increases the distance from the nose to the target; so, longer instruments might be needed to reach the deepest aspect of the nasal cavity. Second, it could take time for users to become accustomed to the mask as it has its learning curve, which could increase surgical times initially. Third, some viral particles could be retained in the valve cover, an aspect that requires further studies. Nevertheless, the mask is meant to be sterilizable and reusable whereas the valve cover is meant to be disposable. Finally, some pressure damage to the facial skin could occur in long procedures; therefore, the mask's posterior rim should be covered with some kind of surgical padded foam and the mask should not be tied tight to the patient's head.

Recommendations have been published in the otolaryngology and skull base surgery literature regarding preprocedure COVID-19 testing, and the use of a powered airpurifying respirator or $\mathrm{N} 95$ mask in addition to PPE. The proposed mask could be useful and offer extra safety if an endoscopic nasal or skull base procedure is required in certain situations: (a) where polymerase chain reaction (PCR) tests are not widely available; (b) in emergencies where two tests cannot be done; (c) in high-risk patients with a negative PCR test, owing to the significant false-negative rate of current reverse transcription PCR assays for COVID-19 $[26,27]$. Moreover, it protects not only surgeons but all the operating room personnel.

Finally, the Maskpirator may also prove useful for otolaryngologists to perform endoscopic in-office procedures, as they return to offer quality care to their patients amid the COVID-19 pandemic [28]. In this respect, it is important to study the tolerance of the conscious patient to the use of the proposed device.

\section{Conclusion}

We have designed and tested a 3D printable facial mask with continuous suction which partially seals the nose when placed in the midfacial area of a patient. This device sucks out gases and aerosols generated during endoscopic endonasal skull base procedures while allowing comfortable handling of endoscopic surgical instruments. The mask is meant to reduce leakage of aerosols in the operating room and decrease the possibility of airborne transmission of viruses among surgical personnel during the COVID-19 pandemic. This device also has potential benefits for reducing longterm exposure to surgical smoke.

Funding No funding was used to support this work.

Data availability All data and videos recorded in this study are available to be analysed.

\section{Compliance with ethical standards}

Conflict of interest The authors declare no existing conflict of interest.

Ethical approval No ethics approval was needed as this was a laboratory study with no patients or animal involvement.

Consent to participate The volunteer in the experiments recorded in video in this study is the second author of the manuscript and he gives his full consent to participate and to be published.

\section{References}

1. Li Q, Guan X, Wu P et al (2020) Early transmission dynamics in Wuhan, China, of novel coronavirus-infected pneumonia. N Engl J Med 382(13):1199-1207. https://doi.org/10.1056/NEJMoa2001 316

2. Organization WH (2020) Advice on the use of masks in the context of COVID-19: interim guidance. 6 April 2020. World Health Organization 
3. Bourouiba L (2020) Turbulent gas clouds and respiratory pathogen emissions: potential implications for reducing transmission of COVID-19. JAMA. https://doi.org/10.1001/jama.2020.4756

4. van Doremalen N, Bushmaker T, Morris DH et al (2020) Aerosol and surface stability of SARS-CoV-2 as compared with SARS-CoV-1. N Engl J Med 382(16):1564-1567. https://doi. org/10.1056/NEJMc2004973

5. Wang W, Xu Y, Gao R et al (2020) Detection of SARS-CoV-2 in different types of clinical specimens. JAMA J Am Med Assoc. https://doi.org/10.1001/jama.2020.3786

6. Judson SD, Munster VJ (2019) Nosocomial transmission of emerging viruses via aerosol-generating medical procedures. Viruses 11(10):940. https://doi.org/10.3390/v11100940

7. Tran K, Cimon K, Severn M et al (2012) Aerosol generating procedures and risk of transmission of acute respiratory infections to healthcare workers: a systematic review. PLoS One 7(4):e35797. https://doi.org/10.1371/journal.pone.0035797

8. Thamboo A, Lea J, Sommer DD et al (2020) Clinical evidence based review and recommendations of aerosol generating medical procedures in otolaryngology-head and neck surgery during the COVID-19 pandemic. J Otolaryngol Head Neck Surg 49(1):1-14. https://doi.org/10.1186/s40463-020-00425-6

9. Zou L, Ruan F, Huang M et al (2020) SARS-CoV-2 viral load in upper respiratory specimens of infected patients. N Engl J Med 382(12):1177-1179. https://doi.org/10.1056/NEJMc2001737

10. Castelnuovo P, Turri-Zanoni M, Karligkiotis A et al (2020) Skullbase surgery during the COVID-19 pandemic: the Italian Skull Base Society recommendations. Int Forum Allergy Rhinol. https ://doi.org/10.1002/alr.22596

11. Workman AD, Welling DB, Carter BS et al (2020) Endonasal instrumentation and aerosolization risk in the era of COVID-19: simulation, literature review, and proposed mitigation strategies. Int Forum Allergy Rhinol. https://doi.org/10.1002/alr.22577

12. Sharma D, Rubel KE, Ye MJ et al (2020) Cadaveric simulation of endoscopic endonasal procedures: analysis of droplet splatter patterns during the COVID-19 pandemic. Otolaryngol Neck Surg Off J Am Acad Otolaryngol Neck Surg 163(1):145-150. https:// doi.org/10.1177/0194599820929274

13. Mayo-Yánez M, Calvo-Henríquez C, Lechien JR, Fakhry $\mathrm{N}$ et al (2020) Is the ultrasonic scalpel recommended in head and neck surgery during the COVID-19 pandemic? State-of-the-art review. Head Neck 42(7):1657-1663. https://doi.org/10.1002/hed.26278

14. Mowbray N, Ansell J, Warren N et al (2013) Is surgical smoke harmful to theater staff? A systematic review. Surg Endosc 27(9):3100-3107. https://doi.org/10.1007/s00464-013-2940-5

15. Kwak HD, Kim S-H, Seo YS, Song K-J (2016) Detecting hepatitis $\mathrm{B}$ virus in surgical smoke emitted during laparoscopic surgery. Occup Environ Med 73(12):857-863. https://doi.org/10.1136/ oemed-2016-103724

16. Kashima HK, Kessis T, Mounts P, Shah K (1991) Polymerase chain reaction identification of human papillomavirus DNA in $\mathrm{CO} 2$ laser plume from recurrent respiratory papillomatosis. Otolaryngol Neck Surg Off J Am Acad Otolaryngol Neck Surg 104(2):191-195. https://doi.org/10.1177/019459989110400206
17. Clarke AL (2020) 3D printed circuit splitter and flow restriction devices for multiple patient lung ventilation using one anaesthesia workstation or ventilator. Anaesthesia. https://doi.org/10.1111/ anae. 15063

18. Viera-Artiles J, Valdiande JJ (2020) 3D-printable headlight face shield adapter. Personal protective equipment in the COVID19 era. United States. 102576. https://doi.org/10.1016/j.amjot o.2020.102576

19. Mick P, Murphy R (2020) Aerosol-generating otolaryngology procedures and the need for enhanced PPE during the COVID19 pandemic: a literature review. J Otolaryngol Head Neck Surg 49(1):1-10. https://doi.org/10.1186/s40463-020-00424-7

20. Lammers MJW, Lea J, Westerberg BD (2020) Guidance for otolaryngology health care workers performing aerosol generating medical procedures during the COVID-19 pandemic. J Otolaryngol Head Neck Surg 49(1):36. https://doi.org/10.1186/s4046 3-020-00429-2

21. Rna Z, Control D, Guiana F et al (2017) SARS-CoV-2 viral load in upper respiratory specimens of infected patients. N Engl J Med 382:1177-1179

22. Kocher GJ, Sesia SB, Lopez-Hilfiker F, Schmid RA (2019) Surgical smoke: still an underestimated health hazard in the operating theatre. Eur J Cardio Thorac Surg 55(4):626-631. https://doi. org/10.1093/ejcts/ezy356

23. Limchantra IV, Fong Y, Melstrom KA (2019) Surgical smoke exposure in operating room personnel: a review. JAMA Surg 154(10):960-967. https://doi.org/10.1001/jamasurg.2019.2515

24. Stanley K (2019) Diathermy smoke shown to be hazardous, so why are we not protecting ourselves? J Perioper Pract 29(10):321327. https://doi.org/10.1177/1750458919877786

25. Mowbray NG, Ansell J, Horwood J et al (2020) Safe management of surgical smoke in the age of COVID-19. Br J Surg. https://doi. org/10.1002/bjs.11679

26. Patel ZM, Fernandez-Miranda J, Hwang PH et al (2020) Letter: Precautions for endoscopic transnasal skull base surgery during the COVID-19 pandemic. Neurosurgery 87(1):E66-E67. https:// doi.org/10.1093/neuros/nyaa125

27. Lynch JB, Davitkov P, Anderson DJ et al (2020) Infectious Diseases Society of America Guidelines on infection prevention for health care personnel caring for patients with suspected or known COVID-19. Clin Infect Dis Off Publ Infect Dis Soc Am. https:// doi.org/10.1093/cid/ciaa1063

28. Narwani V, Kohli N, Lerner MZ (2020) Application of a modified endoscopy face mask for flexible laryngoscopy during the COVID-19 pandemic. Otolaryngol Neck Surg Off J Am Acad Otolaryngol Neck Surg 163(1):107-109. https://doi. org/10.1177/0194599820928977

Publisher's Note Springer Nature remains neutral with regard to jurisdictional claims in published maps and institutional affiliations. 